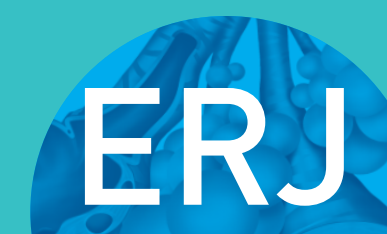

open research
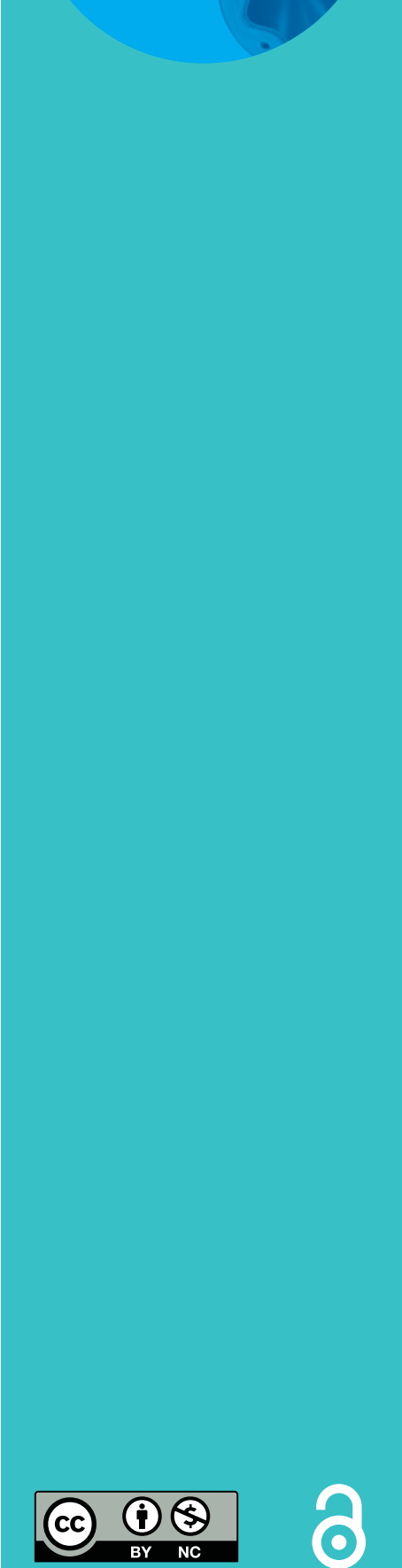

\section{The effects of exogenous lipid on THP-1 cells: an in vitro model of airway aspiration?}

\author{
Yvette A. Hayman, Laura R. Sadofsky, James D. Williamson, Simon P. Hart and \\ Alyn H. Morice
}

Affiliation: Centre for Cardiovascular and Metabolic Research, Hull York Medical School, University of Hull, Hull, UK.

Correspondence: Laura R. Sadofsky, The Wolfson Building, The University of Hull, Cottingham Road, Hull HU6 7RX, UK. E-mail: l.r.sadofskyahull.ac.uk

ABSTRACT Chronic inflammatory diseases of the airways are associated with gastro-oesophageal reflux (GOR) and aspiration events. The observation of lipid-laden macrophages (LLMs) within the airway may indicate aspiration secondary to GOR. The proposed mechanism, that lipid droplets from undigested or partially digested food are aspirated leading to accumulation in scavenging macrophages, led us to hypothesise that an activated population of LLMs could interact with other immune cells to induce bronchial inflammation.

To test this, we generated an in vitro model using differentiated THP-1 cells, which were treated with a high-fat liquid feed.

Here, we show that THP-1 cells can take up lipid from the high-fat feed independent of actin polymerisation or CD36-dependent phagocytosis. These cells did not exhibit M1 or M2 polarisation. Gene array analysis confirmed over 8000 genes were upregulated by at least twofold following high fat exposure, and IL-8 was the most upregulated gene. Pathway analysis revealed upregulation of genes known to be involved in chronic obstructive pulmonary disease (COPD) pathophysiology.

We suggest that aspiration and macrophage phagocytosis may be important mechanisms in the aetiology of diseases such as COPD and cystic fibrosis that are characterised by high levels of IL- 8 within the airways.

@ERSpublications

Lipid treatment of macrophages alters gene expression in pathways known to be involved in COPD pathophysiology http://ow.ly/61pu308T8MM

Cite this article as: Hayman YA, Sadofsky LR, Williamson JD, et al. The effects of exogenous lipid on THP-1 cells: an in vitro model of airway aspiration? ERJ Open Res 2017; 3: 00026-2016 [https:// doi.org/10.1183/23120541.00026-2016].

This article has supplementary material available from openres.ersjournals.com

Received: Feb 222016 | Accepted after revision: Jan 142017

Conflict of interest: None declared.

Copyright $\odot$ ERS 2017. This article is open access and distributed under the terms of the Creative Commons Attribution Non-Commercial Licence 4.0. 


\section{Lessons for clinicians}

- Alveolar macrophages may be able to accumulate dietary lipids following aspiration into the lungs.

- Lipid-laden macrophages display altered gene transcription and could interact with other immune cells to induce bronchial inflammation.

- Thus, aspiration and macrophage phagocytosis may be important mechanisms in the aetiology of diseases including COPD.

\section{Introduction}

Gastro-oesophageal reflux (GOR) is epidemiologically associated with a number of diseases of the airways. It is commonly reported in chronic obstructive pulmonary disease (COPD) exacerbations, cystic fibrosis (CF) and non-CF bronchiectasis, and has been implicated in $\sim 50 \%$ of difficult-to-treat asthma cases $[1,2]$. GOR may be defined by retrograde transport of gastric contents and may comprise liquid alone or more commonly a mixture of liquid and gaseous or particulate reflux [3]. Ascent of stomach contents into the upper airways, pharynx and beyond has been variously labelled as extra-oesophageal reflux, laryngo-pharyngeal reflux or, more recently, airway reflux. Aspiration into the upper and lower airways causes pneumonia, bronchitis and rhinitis, but the mechanism whereby aspiration induces the inflammatory cascade has not previously been addressed.

The inhaled refluxate consists of aerosolised stomach acid, bile, partially digested food matter, bacteria as well as many other constituents including proteolytic enzymes such as pepsin [3-6]. Homogenised lipids are also present in refluxate and can be detected following ingestion by alveolar macrophages using established lipid-staining techniques [7]. A recent study using lipid staining of macrophages from bronchoalveolar lavage fluid found a strong association between lipid-laden macrophages (LLMs) and GOR, but not asthma [8]. The macrophage is capable of scavenging lipid, but the mechanism of fatty acid internalisation and lipid droplet formation is still largely unknown [9-11]. It is recognised that passive diffusion may occur by insertion of the hydrophobic fatty acids into the external surface of the plasma membrane followed by a "flip-flop" mechanism, through which the lipid enters the cytoplasm. By contrast, circulating lipid in the form of low-density lipoprotein (LDL) is known to enter the macrophage via both actin-dependent and -independent mechanisms of endocytosis [12]. Whether this is also true in the case of exogenous, unpackaged fatty acids remains unknown. Macrophages express the class A scavenger receptor and the oxidised LDL scavenger receptor CD36 [13], both of which are capable of binding modified LDL. In the circulation this leads to "foam cell" formation and the arterial wall changes of atherosclerosis.

M1 macrophages are classically activated by bacteria and are usually associated with a cell-mediated inflammatory response [14]. M2 polarised macrophages are alternatively activated and are normally associated with resolution and tissue repair [15]. In atherosclerotic plaques, both classically and alternatively activated cells (M1 and M2, respectively) are present. The polarisation of the macrophage may respond to and modulate different stages of the disease $[16,17]$. In the lung, the presence of lipid-laden alveolar macrophages in sputum or on brochoalveolar lavage has been reported to be indicative of aspiration secondary to GOR [18-21]. Specificity is, however, limited, because intracellular lipid accumulation may occur in other inflammatory processes such as pneumonia. Although studies have been carried out on the mode of uptake of circulating modified LDL in foam cells, alveolar macrophage lipid uptake has so far been overlooked.

We hypothesise that lipid droplets present in airway reflux may be aspirated and accumulate in scavenging alveolar macrophages. Foam cell formation would thus generate activated macrophages, propagating an immune response by the release of inflammatory cytokines, in turn causing airway inflammation and remodelling. Obtaining primary alveolar macrophages can be difficult, so models such as phorbol 12-myristate 13-acetate (PMA) treated THP-1 cells are routinely used and accepted as a satisfactory model [22-24]. Using the human monocytic leukaemia cell line, THP-1, stimulated with PMA to induce monocyte-to-macrophage differentiation [25], we investigated the activation status of LLMs generated by the uptake of unmodified lipid and compared this to classically and alternatively activated macrophages. In addition, we investigated the mode of uptake of unmodified lipid by the macrophage by inhibiting actin-dependent phagocytosis and by blocking the CD36 scavenger receptor. Finally, we elucidated the change in gene expression and cytokine profile consequent on lipid uptake.

\section{Methods}

\section{Cell culture}

THP-1 cells were maintained in RPMI 1640 with $10 \%$ fetal bovine serum, penicillin $\left(100 \mathrm{U} \cdot \mathrm{mL}^{-1}\right)$ and streptomycin $\left(0.1 \mathrm{mg} \cdot \mathrm{mL}^{-1}\right)$ (Gibco, Paisley, UK). Cells $\left(0.5 \times 10^{6}\right.$ cells $\left.\cdot \mathrm{mL}^{-1}\right)$ were differentiated in medium with $50 \mathrm{nM}$ PMA $(24 \mathrm{~h})$ then PMA-free medium $(24 \mathrm{~h})$ before further treatments. Differentiation (PMA/THP-1) was determined by cell adherence, increased nucleus-to-cytoplasm ratio, and mitotic growth inhibition. 


\section{Oil Red 0 staining}

PMA/THP-1 cells were incubated with 10\% Calogen, the medical high-fat liquid food (Nutricia Advanced Medical Nutrition, Wiltshire, UK) for $24 \mathrm{~h}$. Staining of intracellular lipid droplets was performed using the Oil Red O (ORO) stain. This stain is more soluble in lipid than in aqueous solvent, staining nonpolar lipids a bright red colour. A stock solution of ORO (0.5\% in isopropanol) was diluted and filtered $(60 \%$ stock solution and $40 \%$ distilled water). Slides were stained $(20 \mathrm{~min})$ and counterstained $(3 \mathrm{~min})$ with Mayer's haematoxylin followed by two to three drops of $0.1 \%$ sodium bicarbonate $(30 \mathrm{~s})$. Slides were dried and mounted using Kaiser's glyceringelatine mounting medium.

\section{Assessment of phagocytosis}

PMA/THP-1 cells were incubated with $1 \mathrm{mg} \cdot \mathrm{mL}^{-1}$ zymosan $(1 \mathrm{~h})$ and stained using a Reastain Quick-Diff Kit (Reagena Ltd, Toivala, Finland). Phagocytosed zymosan appeared as white rings in the cellular cytoplasm and was quantified using the phagocytic index.

PMA/THP-1 cells were incubated in medium containing $10 \mu \mathrm{g} \cdot \mathrm{mL}^{-1}$ cytochalasin $\mathrm{D}$ or mouse monoclonal CD36 clone FA6-152 blocking antibody $\left(1 \mu \mathrm{g} \cdot \mathrm{mL}^{-1}\right.$; Santa Cruz, Heidelberg, Germany) for $1 \mathrm{~h}$ before incubation with zymosan $(1 \mathrm{~h})$ or Calogen $(4 \mathrm{~h})$. Coverslips were then differentially stained or stained with ORO.

\section{Transmission electron microscopy}

Cells were harvested then fixed in iso-osmotic $2.5 \%$ glutaraldehyde diluted in sodium cacodylate buffer $(\mathrm{pH}$ 7.3) and post-fixed in $1 \%$ osmium tetroxide. Cells were then stained with $1 \%$ uranyl acetate and dehydrated through an ethanol series and eventually propylene oxide. Cells were embedded in epon resin and ultrathin sections were cut. Images were obtained in a Jeol 2010 transmission electron microscope (TEM) running at $120 \mathrm{kV}$ using a Gatan US4000 digital camera.

\section{Gene microarray}

mRNA (duplicates of four) was extracted using a NucleoSpin ${ }^{\circ}$ RNA II Kit (Macherey-Nagel, Duren, Germany) and quantified on a Qubit ${ }^{\mathrm{TM}}$ fluorometer (Invitrogen, Paisley, UK) before gene expression profiling and analysis (Bioscience Technology Facility, University of York), using an Agilent gene array system. Data were analysed through the use of QIAGEN's Ingenuity Pathway Analysis (IPA, QIAGEN Redwood City, www.qiagen.com/ingenuity).

\section{ELISAS}

PMA/THP-1 cells were either left untreated or treated to induce M1 (lipopolysaccharide, $10 \mathrm{ng} \cdot \mathrm{mL}^{-1}$ ) or M2 (IgG, $150 \mathrm{ng} \cdot \mathrm{mL}^{-1}$ or M-CSF, $50 \mathrm{ng} \cdot \mathrm{mL}^{-1}$ ) cell polarisation. Release of IL-4, IL-8, IL-12 and $\beta$-NGF was measured by ELISA (R\&D Systems, Abingdon) (detection range $31.2-2000 \mathrm{pg} \cdot \mathrm{mL}^{-1}$ for all kits). Plates were analysed on a Multiskan FC plate-reader (Thermo Scientific) at $450 \mathrm{~nm}$, subtracting readings at $570 \mathrm{~nm}$ to correct for plate optical imperfections.

\section{Flow cytometry}

Cells were harvested, counted and filtered through a $70 \mu \mathrm{m}$ cell strainer (BD Biosciences), and transferred to 96 -well tissue culture plates at a concentration of $1 \times 10^{5}$ cells per well. The plate was centrifuged $(350 \mathrm{~g}$, $3 \mathrm{~min}, 4^{\circ} \mathrm{C}$ ) and washed twice with PBS before incubating $\left(30 \mathrm{~min}\right.$ at $4{ }^{\circ} \mathrm{C}$ ) with mouse monoclonal primary antibodies (CD11b, CD14, CD16, CD23, CD24, CD32, CD36, CD64, CD80, CD86, CD163, CD206 $\left(20 \mu \mathrm{g} \cdot \mathrm{mL}^{-1}, \mathrm{AbD}\right.$ Serotec, Oxford, UK) or CD32 [26]). Cells were washed with PBS and centrifuged (350g, $5 \mathrm{~min}, 4^{\circ} \mathrm{C}$ ) twice and the supernatant discarded. The cells were then incubated with fluorescein isothiocyanate (FITC)-conjugated rabbit anti-mouse IgG secondary antibody $\left(50 \mu \mathrm{g} \cdot \mathrm{mL}^{-1}\right.$, Sigma Aldrich, Poole, UK) with gentle agitation ( $30 \mathrm{~min}$ at $4{ }^{\circ} \mathrm{C}$ in the dark). The cells were washed as previously described and re-suspended in $500 \mu \mathrm{L}$ FACSFlow Buffer (BD Biosciences) before analysis on a FACSAria II flow cytometer (BD Biosciences) running BD FACSDiva version 6.1.3 software and counting $1 \times 10^{4}$ cells per sample. During analysis, a gate (P1) was applied to the live cell population based on forward side-scatter characteristics. This gate was applied to a histogram of fluorescence to all subsequent samples within the same experiment, and statistical data were generated to include mean fluorescence intensities.

\section{Statistical analysis}

Data are expressed as means \pm SEM. For the assessment of phagocytosis, an unpaired, two-tailed t-test was performed for samples with unequal variance (Microsoft Excel 2010). An independent sample Kruskal-Wallis test was performed to highlight significant differences in cell surface expression following treatments, followed by Mann-Whitney testing (SPSS v19; IBM, Armonk, NY, USA). For the gene 
microarray analysis, significance is displayed as the p-values corrected (p-value (corr)) for multiple testing using the Benjamini-Hochberg FDR (false discovery rate) method.

\section{Results}

\section{Assessment of phagocytosis}

Initially, we sought to determine whether PMA-differentiated THP-1 cells accumulated lipid from Calogen, a medical high-fat liquid feed. Indeed, PMA-differentiated THP-1 cells were able to take up lipid following exposure to Calogen (figure $1 \mathrm{a}$ and b). Next we investigated possible mechanisms of lipid uptake, either via phagocytosis or CD36 receptor mediated lipid uptake. Cytochalasin D, a fungal metabolite, binds to actin filaments and inhibits actin polymerisation, thus inhibiting actin dependent phagocytosis. CD36 is a scavenger receptor which is involved in lipid uptake and foam cell formation. In order to assess whether this receptor was involved in lipid uptake in our model, we inhibited CD36 using a monoclonal CD36 blocking antibody. Pre-incubation with cytochalasin D and CD36 blocking antibody failed to inhibit lipid uptake by PMA/THP-1 cells (figure 1c and d) despite the cytochalasin D inhibiting zymosan uptake by these cells (supplementary figure S1). These cells were visualised under TEM (figure 1e). Identified lipid droplets were then magnified for closer inspection (figure 1f and g). Lipid droplets were visible randomly throughout the cytoplasm and were identifiable by dark staining of residual lipid remaining after the fixing process. These droplets could be differentiated from cholesterol clefts formed by crystallisation of cholesterol during the fixation procedure, visible as long clear spaces in the cytoplasm. Closer inspection revealed what appears to be a double membrane surrounding the lipid droplet which was variable in size and shape although roughly rounded.

\section{Gene microarray data analysis}

Uptake of lipids by macrophages is known to initiate a signalling cascade within the cells, which may initiate an inflammatory response. We therefore used gene microarrays to compare gene expression preand post-lipid exposure in PMA-differentiated THP-1 cells. This was followed by pathway analysis to identify possible signalling pathways induced by the lipid uptake. Microarray data are available in the
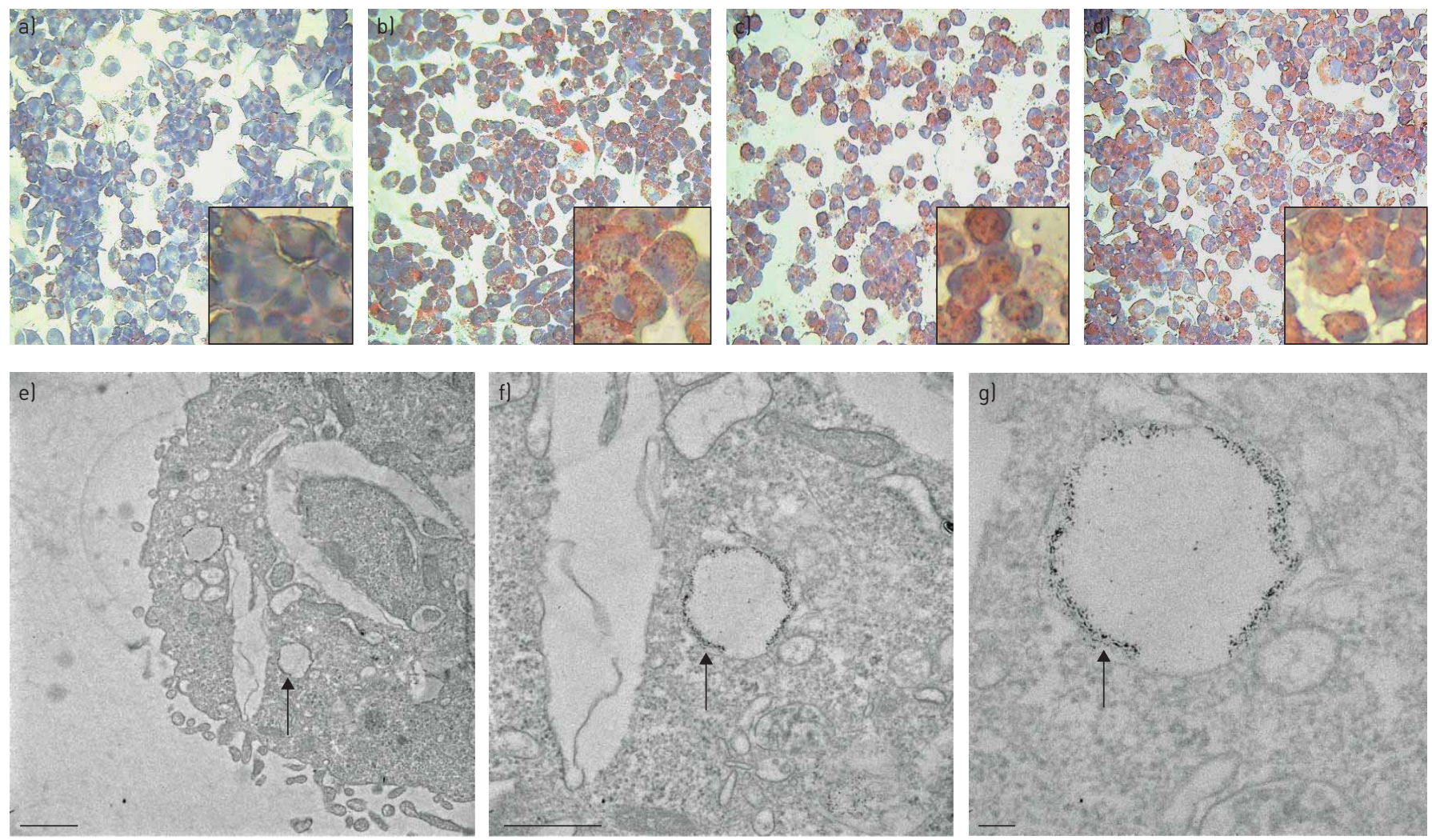

FIGURE 1 PMA/THP-1 lipid uptake. PMA/THP-1 cells were incubated with 10\% vol/vol Calogen for $24 \mathrm{~h}$ with and without inhibitors and were stained with ORO to detect lipid accumulation. a) Untreated cells were compared with b) cells treated with Calogen alone and c) in the presence of cytochalasin D and d) CD36 monoclonal blocking antibody. Inset images are magnified representative regions of each treatment. e) Cells treated with Calogen were also visualised under TEM. Scale bar $=1 \mu \mathrm{m}$. f, g) Lipid droplets were identified and magnified to reveal dark stained remnants of lipid encased in membrane-bound vesicles, as indicated by the arrows. Scale bars=500 nm (f) and $100 \mathrm{~nm}(\mathrm{~g})$. 
ArrayExpress database (www.ebi.ac.uk/arrayexpress) under accession number E-MTAB-4153. PMA treatment of THP-1 cells induced 8130 genes to be upregulated or downregulated by twofold or more. Upon Calogen stimulation of PMA/THP-1 cells, 8502 genes showed fold change differences of two or more compared with PMA treatment alone. The majority of these genes (5396) showed two- to threefold differences. Table 1 shows the top 10 upregulated and downregulated genes for Calogen-treated PMA/ THP-1 cells compared to PMA/THP-1 cells alone. Interestingly, in addition to these genes, IL-1 $\beta$ was also upregulated 32-fold following lipid treatment. The top 10 upregulated and downregulated genes in PMA/ THP-1 cells compared to THP-1 cells alone are shown in supplementary table S1 (note that some genes are probed for twice, including IL-8 and CCL4). Gene functions were adapted from the NCBI gene database and the GeneCards human gene database index.

TABLE 1 Top 10 up- and downregulated genes following Calogen treatment of PMA/THP-1 cells

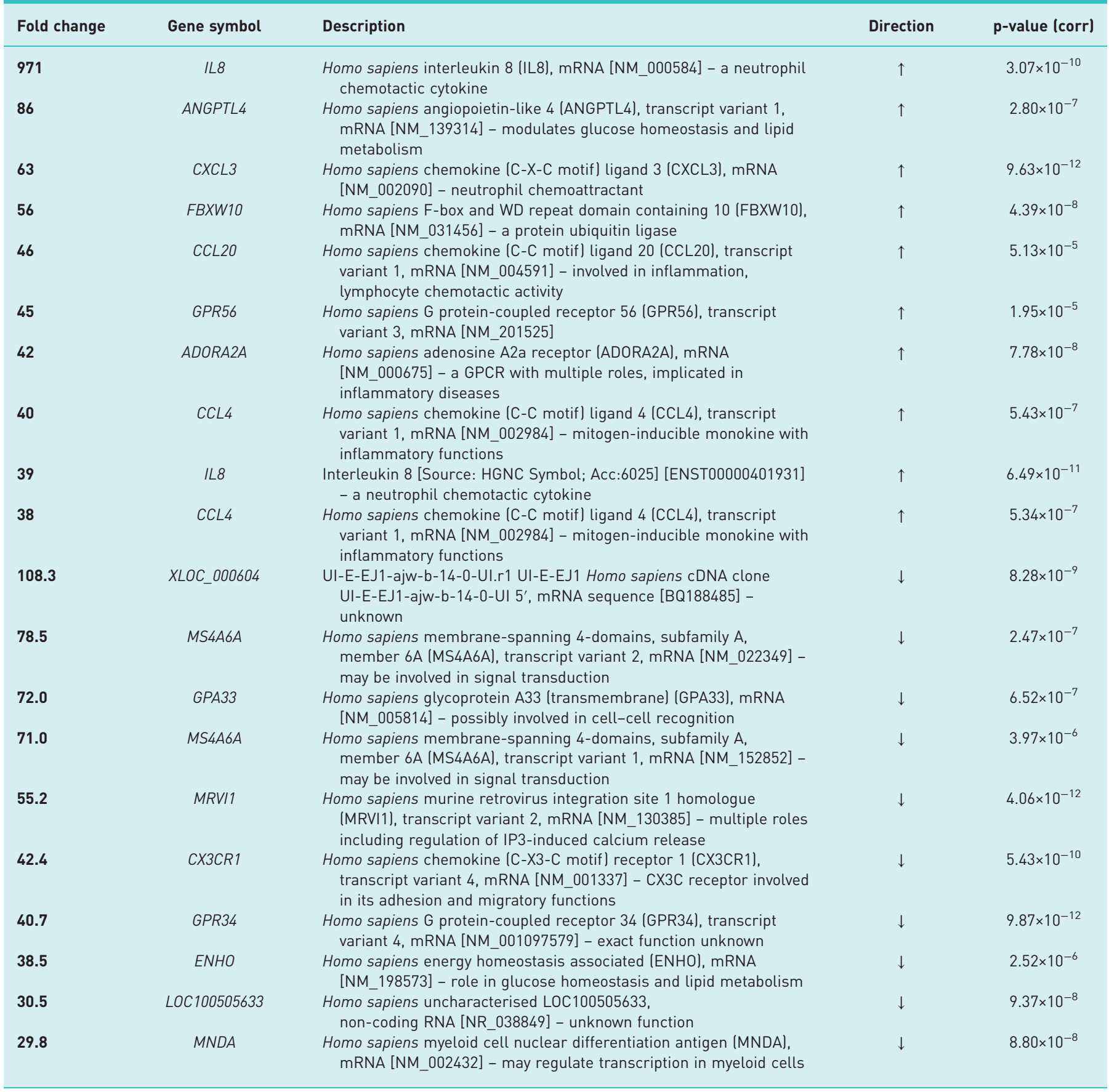


Data were analysed through the use of QIAGEN's Ingenuity Pathway Analysis (IPA) (QIAGEN, Redwood City, CA, USA), which revealed that Calogen treatment of PMA/THP-1 cells induced an increase in expression in a number of genes important in the development of COPD (figure 2). These genes include IL- 8, TNF- $\alpha$, CXCL2 and MMPs 8 and 1.

Analysis of the gene array data for markers of macrophage polarisation, following Calogen treatment, showed a trend towards M2 polarisation, with increases in IL-8 and CD23 genes and decreases in M1 markers (CD16, CD32 and CD64 genes).

\section{ELISA cytokine analysis}

Released cytokines were measured using ELISAs to determine whether the lipid-treated PMA/THP-1 cells displayed an M1 or M2 phenotype. PMA/THP-1 cells were either left untreated or treated to induce M1 (PMA or LPS) or M2 (IgG or M-CSF) cell polarisation. IL-4 production was used to detect M2 macrophage polarisation. Concentrations of IL-4 were detected at very low levels (around $100 \mathrm{pg} \cdot \mathrm{mL}^{-1}$ ) in all supernatants taken from THP-1 cell cultures, with no significant difference between treatments (figure 3a).

Very low levels of the neutrophil chemoattractant protein, IL-8, were detected in untreated THP-1 cell culture samples. In samples taken from PMA/THP-1 cell culture, IL-8 protein concentrations were significantly higher following all treatments (figure $3 \mathrm{~b}$ ). Treatment with Calogen did not further increase IL-8 secretion and, although there was a slight increase in Calogen-induced IL-4 secretion, this was not significant. Levels of both IL-12, the M1 macrophage marker, and NGF, were undetectable (data not shown).

\section{Immunophenotype of polarised and LLMS}

THP-1 cells were left untreated or stimulated to differentiate with PMA. PMA/THP-1 cells were then stimulated to become M1 or M2 polarised activated macrophages with either LPS (M1), heat-aggregated human IgG (M2) or M-CSF (M2, data not shown). PMA/THP-1 cells were also treated with Calogen before comparing key cell surface markers (supplementary table S2 gives a description of each marker), known to be indicators of M1 or M2 polarised macrophages (figure S2), by flow cytometry. Compared with THP-1 cells treated with PMA only, PMA plus LPS-treated cells were not significantly different. Cells treated with PMA plus heat-aggregated human IgG differed from cells treated only with PMA by a significantly decreased expression of CD32 only. This reduction was not seen in M-CSF-treated PMA/ THP-1 cells, which displayed a significantly lower expression of CD163 when compared with PMA-only-treated cells (data not shown). Calogen-treated cells also showed no significant difference when compared with PMA-only-treated cells. When compared with PMA plus LPS-treated cells, Calogen-treated

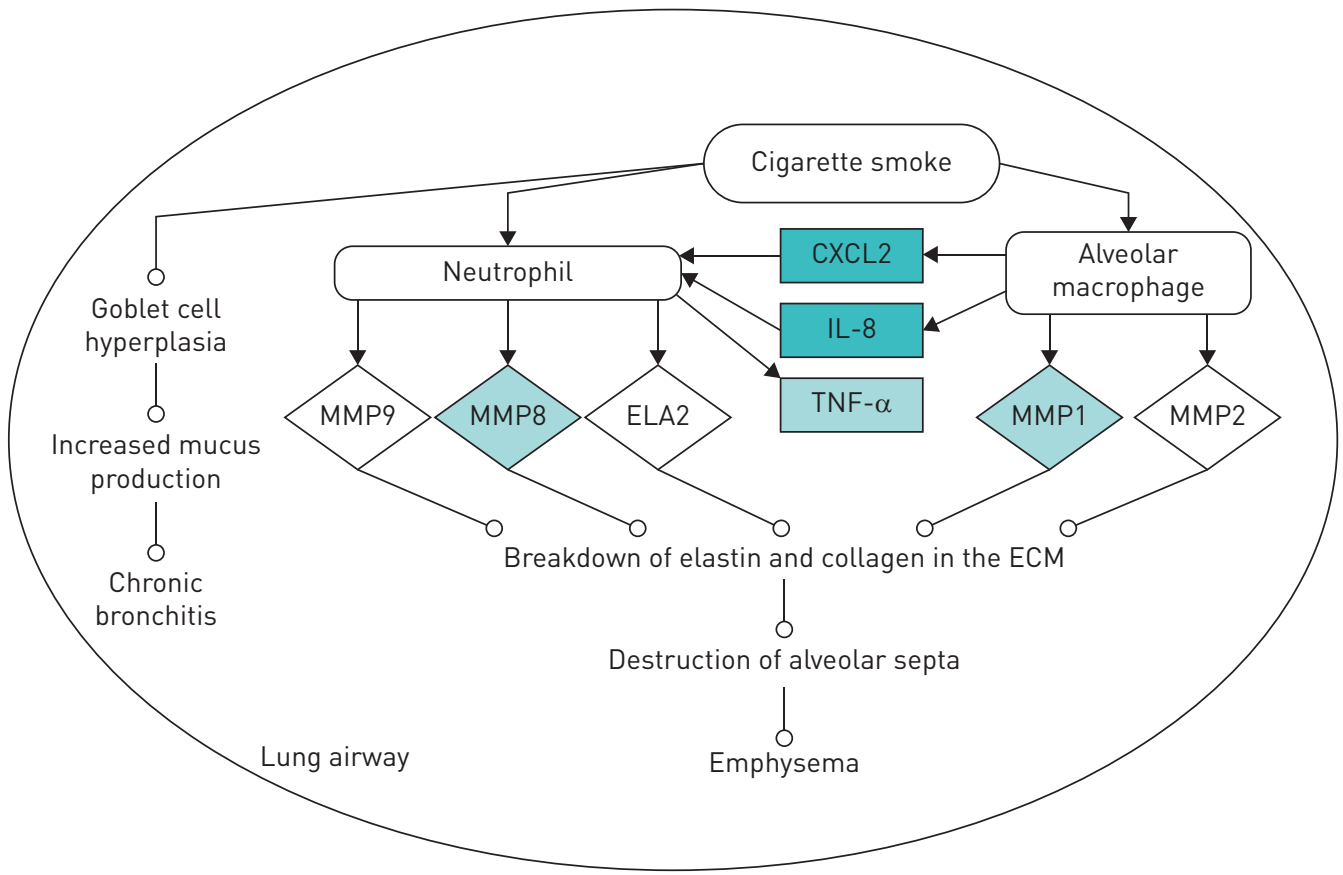

FIGURE 2 Gene microarray pathway analysis. The analysis revealed that Calogen treatment of PMA/THP-1 cells caused upregulation (shaded boxes) of genes associated with the development of COPD (the network was generated using QIAGEN's Ingenuity Pathway Analysis (IPA); QIAGEN, Redwood City, CA, USA). 

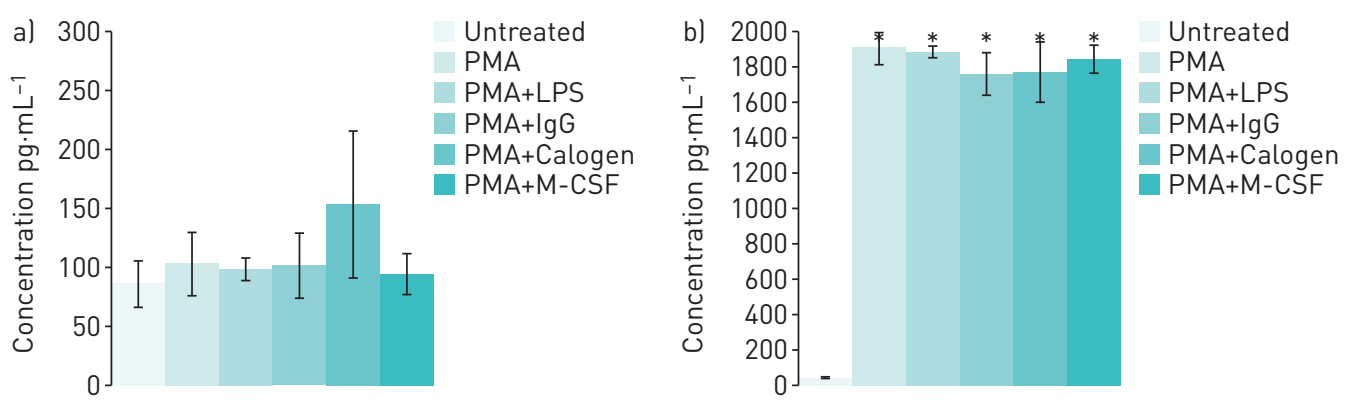

FIGURE 3 IL-4 and IL-8 secretion by THP-1 cells. Concentrations of a) IL-4 and b) IL-8 protein detected by ELISA in cell culture supernatants of THP-1 cells subjected to a variety of treatments. PMA: phorbol 12-myristate 13-acetate; LPS: lipopolysaccharide. *: $p<0.05$.

cells showed significantly higher CD14 expression and lower CD32 expression. Finally, when compared with PMA plus heat-aggregated human IgG-treated cells, Calogen-treated cells were not different.

\section{Discussion}

Alveolar macrophages make up $\sim 70 \%$ of the immune cells populating the airway and are capable of antigen presentation and modulation of the immune response. The alveolar macrophage is also capable of lipid uptake, which may activate and induce an inflammatory phenotype by mechanisms currently unknown. We hypothesised that lipid droplets from aerosolised gastric contents, when aspirated into the airway, accumulate in scavenging macrophages. LLM formation in the airway may thus generate activated macrophages that interact with other immune cells and tissue to induce an airway inflammation and fibrosis characteristic of a number of different respiratory conditions.

PMA/THP-1 incubated with $10 \%$ vol/vol Calogen were stained using ORO and showed intense lipid staining. This staining is probably due to the direct uptake of lipids from the Calogen, as little or no staining was detected in the PMA/THP-1-only cells. Calogen was used in this study because of the high standard of manufacturing process used in products for human consumption, and because aspiration of Calogen feed is a particularly common clinical scenario during nasogastric and enteral feeding. Calogen contains a combination of sunflower oil and canola oil, which comprise varying combinations of both long-chain unsaturated (89.4\%) and long-chain saturated (10.6\%) fatty acids, along with a small amount of trans fats.

Upon visualisation by TEM, accumulated lipid droplets were separate from, and in addition to, cholesterol clefts. They appeared to be randomly distributed throughout the cytoplasm, bearing no obvious association to specific organelles or structures, suggesting passive diffusion through the cell membrane rather than the previously hypothesised endogenous production from the endoplasmic reticulum [9].

In order to decipher the mechanism by which lipids can be internalised by macrophages, we inhibited actin-dependent phagocytosis by incubation with cytochalasin D. Cytochalasin D is a fungal metabolite that binds actin filaments to prevent polymerisation. When PMA/THP-1 were pre-incubated with cytochalasin D before incubation with Calogen, lipid uptake was unaffected. Cells were also pre-incubated with a commercially available antibody clone capable of blocking CD36. This also appeared to have no effect on lipid accumulation. There are a number of different ways for macrophages to take up lipids (summarised by [27]). In this study we were unable to identify the exact mechanism; however, our results suggest that internalisation of lipids is actin-independent and in agreement with the hypothesis that fatty acids can diffuse across cells membranes. The TEM pictures showed that the lipids were present within the cytosol and appeared to be surrounded by a double membrane that could indicate autophagy. Once inside the cell, the lipids may be encapsulated, creating autophagosomes that are degraded by lysosomes [28].

Upon Calogen stimulation, 8502 genes were upregulated twofold or greater in PMA/THP-1 cells. The majority of these genes (5398) were two- to threefold different. The present results indicate that lipid uptake has a direct effect on cell mRNA transcription and may provide clues as to potential therapeutic manipulation of this process. Pathway analysis revealed that the Calogen treatment of PMA/THP-1 cells upregulated genes involved in the development of airway inflammation and fibrosis. Our findings suggest future experimental approaches into the pathophysiology of airways disease resulting from macrophage lipid accumulation.

IL-8 was probed for and detected in all samples by ELISA. In untreated THP-1 cell culture supernatant, levels of IL-8 were particularly low. However, when THP-1 cells were stimulated with PMA, IL-8 levels were significantly raised, regardless of the secondary stimulation. It is possible therefore that PMA stimulation of THP-1 cells causes IL-8 secretion, which masks any further secretion induced by a secondary stimulant. 
Indeed PARK et al. showed that similar PMA concentrations induced IL-8 mRNA upregulation [29]. As neutrophilic infiltration of the airways is a clinical feature of many non-atopic inflammatory airways diseases, including those induced by infection, this finding was perhaps not surprising. However, in our experiments, at the mRNA level IL- 8 was most upregulated in Calogen-treated PMA/THP-1 cells. IL- 8 is known to be prestored in other cell types including endothelial cells [30], and this may also be true for monocytes and macrophages. We thus suggest that, on PMA exposure, intracellular stores are depleted and no further release can be seen with other stimulants such as Calogen. At the mRNA level, however, Calogen causes an upregulation of IL-8 message contributing to replenishment of the intracellular stores. This is a novel mechanism whereby neutrophilic airway inflammation, such as occurs in exacerbations of COPD and "neutrophilic asthma", may be generated in the absence of an infectious stimulus.

We generated in vitro cell cultures of activated macrophages polarised to both the M1 inflammatory phenotype (using LPS) and the M2 anti-inflammatory phenotype (using heat-aggregated human IgG or $\mathrm{M}-\mathrm{CSF}$ ) in order to compare with the LLMs generated by Calogen treatment. On stimulation with heat-aggregated human IgG, we expected PMA/THP-1 cells to polarise to an anti-inflammatory M2 macrophage phenotype with increased expression of the IgE receptor (CD23), upregulation of the haemoglobin and mannose receptors (CD163 and CD206) and possibly with increased expression of CD86, the co-stimulatory molecule required for $\mathrm{TH} 2$ cell differentiation. Heat-aggregated human IgG-treated PMA/THP-1 cells showed only a significant decrease in CD32 compared with PMA-only treated cells, which does not indicate cell polarisation. For this reason we compared heat-aggregated human IgG stimulation with an alternative method for inducing M2 polarisation, namely M-CSF (data not shown). M-CSF stimulation failed to significantly alter CD32 expression compared with PMA-only cells. This would suggest a blocking effect seen by heat-aggregated human IgG, but no effect with M-CSF. A small but significant reduction in CD163 was observed with M-CSF treated cells compared with controls, which would indicate that both heat-aggregated human IgG and M-CSF were ineffective at generating M2 polarised macrophages. In addition, treatment with Calogen did not appear to significantly change the immunophenotype of the PMA/THP-1 cells and thus could not be labelled as M1 or M2 polarised according to recently published markers [17, 31-33]. We were also unable to detect macrophage polarisation by measuring released cytokines. This is perhaps unsurprising, as it appears that macrophages display plasticity and can rarely be classified into two distinct subsets [16].

Although an M2 macrophage polarisation could not be distinguished, we were encouraged by the IL-4 ELISA results produced. Although not significant here, there was a trend to an increase in IL-4 with Calogen treatment, suggesting polarisation may be directed more towards M2. This is also true of IL-8, another M2 marker, which, although not significantly different on ELISA, was highly upregulated on an mRNA level, as determined by gene array analysis.

In conclusion, macrophages are able to accumulate dietary lipids in membrane-bound vesicles distributed throughout the cell cytoplasm. This lipid uptake directly alters gene transcription, including upregulation of IL-8, TNF- $\alpha$, CXCL2 and MMPs 8 and 1, which are involved in inflammatory conditions including COPD.

\section{Acknowledgements}

We would like to thank Paul Kaye and Peter Ashton at the University of York (York, UK) for their help with the gene array analysis.

\section{References}

1 Mathew JL, Singh M, Mittal SK. Gastro-oesophageal reflux and bronchial asthma: current status and future directions. Postgrad Med J 2004; 80: 701-705.

2 Pacheco-Galván A, Hart SP, Morice AH. Relationship between gastro-oesophageal reflux and airway diseases: the airway reflux paradigm. Arch Bronconeumol 2011; 47: 195-203.

3 Balaji NS, Blom D, DeMeester TR, et al. Redefining gastroesophageal reflux (GER). Surg Endosc 2003; 17: $1380-1385$.

4 Morehead RS. Gastro-oesophageal reflux disease and non-asthma lung disease. Eur Respir Rev 2009; 18: 233-243.

5 Oh DS, Hagen JA, Fein M, et al. The impact of reflux composition on mucosal injury and esophageal function. J Gastrointest Surg 2006; 10: 787-796.

6 Johnston N, Wells CW, Samuels TL, et al. Pepsin in nonacidic refluxate can damage hypopharyngeal epithelial cells. Ann Otol Rhinol Laryngol 2009; 118: 677-685.

7 Midulla F, Strappini PM, Ascoli V, et al. Bronchoalveolar lavage cell analysis in a child with chronic lipid pneumonia. Eur Respir J 1998; 11: 239-242.

8 Gibeon D, Zhu J, Sogbesan A, et al. Lipid-laden bronchoalveolar macrophages in asthma and chronic cough. Respir Med 2014; 108: 71-77.

9 Hamilton JA, Kamp F. How are free fatty acids transported in membranes? Is it by proteins or by free diffusion through the lipids? Diabetes 1999; 48: 2255-2269.

10 Zakim D. Thermodynamics of fatty acid transfer. J Membr Biol 2000; 176: 101-109.

11 Glatz JF, Luiken JJ, Bonen A. Membrane fatty acid transporters as regulators of lipid metabolism: implications for metabolic disease. Physiol Rev 2010; 90: 367-417. 
12 Shashkin P, Dragulev B, Ley K. Macrophage differentiation to foam cells. Curr Pharm Des 2005; 11: 3061-3072.

13 Moore KJ, Freeman MW. Scavenger receptors in atherosclerosis: beyond lipid uptake. Arterioscler Thromb Vasc Biol 2006; 26: 1702-1711.

14 Adamson S, Leitinger N. Phenotypic modulation of macrophages in response to plaque lipids. Curr Opin Lipidol 2011; 22: 335-342.

15 Mantovani A, Garlanda C, Locati M. Macrophage diversity and polarization in atherosclerosis: a question of balance. Arterioscler Thromb Vasc Biol 2009; 29: 1419-1423.

16 Mosser DM, Edwards JP. Exploring the full spectrum of macrophage activation. Nat Rev Immunol 2008; 8: 958-969.

17 Bouhlel MA, Derudas B, Rigamonti E, et al. PPAR $\gamma$ activation primes human monocytes into alternative M2 macrophages with anti-inflammatory properties. Cell Metab 2007; 6: 137-143.

18 Ahrens P, Noll C, Kitz R, et al. Lipid-laden alveolar macrophages (LLAM): a useful marker of silent aspiration in children. Pediatr Pulmonol 1999; 28: 83-88

19 Parameswaran K, Anvari M, Efthimiadis A, et al. Lipid-laden macrophages in induced sputum are a marker of oropharyngeal reflux and possible gastric aspiration. Eur Respir J 2000; 16: 1119-1122.

20 Adams R, Ruffin R, Campbell D. The value of the lipid-laden macrophage index in the assessment of aspiration pneumonia. Aust N Z J Med 1997; 27: 550-553.

21 Kazachkov MY, Muhlebach MS, Livasy CA, et al. Lipid-laden macrophage index and inflammation in bronchoalveolar lavage fluids in children. Eur Respir J 2001; 18: 790-795.

22 Groot-Kormelink PJ, Fawcett L, Wright PD, et al. Quantitative GPCR and ion channel transcriptomics in primary alveolar macrophages and macrophage surrogates. BMC Immunol 2012; 13: 57.

23 Weiden M, Tanaka N, Qiao Y, et al. Differentiation of monocytes to macrophages switches the Mycobacterium tuberculosis effect on HIV-1 replication from stimulation to inhibition: modulation of interferon response and CCAAT/enhancer binding protein beta expression. J Immunol 2000; 165: 2028-2039.

24 Macdonald SH, Woodward E, Coleman MM, et al. Networked T cell death following macrophage infection by Mycobacterium tuberculosis. PLoS ONE 2012; 7: e38488.

25 Auwerx J. The human leukemia cell line, THP-1: a multifacetted model for the study of monocyte-macrophage differentiation. Experientia 1991; 47: 22-31.

26 Greenman J, Hogg N, Nikoletti S, et al. Comparative efficiencies of bispecific $\mathrm{F}\left(\mathrm{ab}^{\prime} \gamma\right)_{2}$ and chimeric mouse/human IgG antibodies in recruiting cellular effectors for cytotoxicity via $\mathrm{Fc}$ gamma receptors. Cancer Immunol Immunother 1992; 34: 361-369.

27 Moore KJ, Sheedy FJ, Fisher EA. Macrophages in atherosclerosis: a dynamic balance. Nat Rev Immunol 2013; 13: 709-721.

28 Nakahira K, Pabon Porras MA, Choi AM. Autophagy in pulmonary diseases. Am J Respir Crit Care Med 2016; 194: 1196-1207.

29 Park EK, Jung HS, Yang HI, et al. Optimized THP-1 differentiation is required for the detection of responses to weak stimuli. Inflamm Res 2007; 56: 45-50.

30 Utgaard JO, Jahnsen FL, Bakka A, et al. Rapid secretion of prestored interleukin 8 from Weibel-Palade bodies of microvascular endothelial cells. J Exp Med 1998; 188: 1751-1756.

31 Stout RD, Suttles J. Functional plasticity of macrophages: reversible adaptation to changing microenvironments. J Leukoc Biol 2004; 76: 509-513.

32 Porcheray F, Viaud S, Rimaniol AC, et al. Macrophage activation switching: an asset for the resolution of inflammation. Clin Exp Immunol 2005; 142: 481-489.

33 Anderson CF, Mosser DM. A novel phenotype for an activated macrophage: the type 2 activated macrophage. J Leukoc Biol 2002; 72: 101-106. 